\title{
THE SURGERY OF INFLAMMATORY BOWEL DISEASE
}

\author{
LIEUTENANT COLONEL M S OWEN-SMITH, MS, FRCS \\ Royal Army Medical College, London
}

Inflammatory diseases of the bowel include Crohn's disease, Ulcerative colitis, Diverticulitis, Ischaemic and radiation colitis, specific protozoal and bacterial infections.

The term "Inflammatory bowel disease" has been used to cover the first three of these conditions and today, I, like the other speakers, will cover Crohn's disease and Ulcerative colitis.

\section{Crohn's disease}

Surgically, one of the most important pathological changes is the presence of crypt abscesses. These deep ulcers penetrate into the muscle layers of the gut causing a transmural inflammatory reaction which, in turn, causes the characteristic thickening of the small gut in Crohn's so aptly described as feeling like "an eel in rigor mortis". Other pathological consequences that have surgical significance are stenosis which may lead to sub-acute obstruction, particularly in narrow parts of the gut such as the terminal ileum, the rectum and anastomoses, and finally fistulae which occur when coils of inflamed gut adhere together or when a mobile loop adheres to the peritoneal surface of an abdominal wound or to other viscera.

\section{Surgical management}

Surgery may be required as an emergency or as an elective operation.

\section{Emergency surgery}

Occasionally the abdiomen is opened in a child or young adult because of suspected acute appendicitis. The appendix is found to be normal but a loop of small bowel is characteristically thickened, oedematous and inflamed and a diagnosis of acute Crohn's disease can be made. In the past, appendicectomy has been avoided in such patients because of the fear of producing external fistula but it is far better to remove the appendix so that in the future one is assured that a life threatening acute appendicitis is not present. There is just as much chance of a fistula forming from the diseased segment of small bowel even if the patient is simply closed up.

Emergency operation may be required rarely for a free perforation but quite commonly a perforation results in the formation of an internal abscess and this may require operation. Severe bleeding is rare and stenosis tends to cause subacute rather than acute obstruction. Abscesses are a relatively common complication of Crohn's disease. They occur in the abdominal wall after operation and also many small abscesses may form between adjacent loops of small bowel. This is probably the mechanism leading to eventual entero-enteric fistulae. 
Elective operation

Indications for surgery as an elective operation are local complications such as fistula or abscess, chronic blood and protein loss from active mucosal ulceration, chronic obstructive symptoms or recurrent perianal disease. In most cases in chronic Crohn's the indications for operation are usually multiple and vary in small bowel and large bowel (Table I).

Table I

Indications for operation (After Alexander-Williams ${ }^{10}$ )

\begin{tabular}{|l|c|c|}
\hline & $\begin{array}{c}\text { Small bowel } \\
\text { (percentage) }\end{array}$ & $\begin{array}{c}\text { Large bowel } \\
\text { (percentage) }\end{array}$ \\
\hline Abscess or fistula & 25 & 2 \\
\hline Chronic ill health & 15 & 70 \\
\hline Obstructive symptoms & 60 & 12 \\
\hline Gross perianal disease & 0.5 & 15 \\
\hline
\end{tabular}

Fistula is a common complication of Crohn's. Setting aside anal fistulae, most fistulae derive from disease of small bowel. External fistulae to the skin usually occur in the scar of an operation and may complicate 10 to 50 per cent of all operations for Crohn's disease. Such fistulae rarely close spontaneously and probably excision of the diseased segment of the bowel from which the fistula arises is the best surgical treatment. Steinberg et $a l^{1}$ showed that resections for fistulae were successful in 84 per cent with a six per cent mortality. Internal fistulae may be into other parts of the gut or into the genito-urinary tract. Those between the upper and lower parts of the gut may be associated with sudden deterioration in the patient.

\section{Chronic blood and protein loss. Obstruction}

The problems at openation are whether to by-pass or to resect the involved segment. If resection is decided upon, how much of the bowel should be excised? In general, resection is to be preferred to by-pass operations. The resectionists are of two schools, first those who only resect the segment of gut giving rise to the particular complication which enforced operation. Secondly, those who resect widely taking $25 \mathrm{~cm}$ of apparently normal bowel on either side of the affected segment and removing the enlarged lymphatic glands. The ideal lesion for resection is one that is well localised, which has not progressed and has remained quiescent for many months. By-pass operation, that is short circuit with exclusion, is confined by those surgeons who perform this operation to those patients in whom the findings suggest that resection would be unduly hazardous. Some would also divide the ileum $20 \mathrm{~cm}$ above the diseased segment and do an ileo-transverse colostomy. The operative montality for resection is about two per cent and the recurrence rate nearly 20 per cent at five years and 33 per cent at 10 years $\left(\mathrm{Kyll}^{2}\right)$. Short circuit with exclusion is not such a good procedure, with a recurrence which is higher than resection at 50 per cent. 
Large bowel disease (Lockhart-Mummery3)

About a third of all cases of Crohn's disease involve the large bowel (17 per cent large bowel alone, 17 per cent large bowel and small bowel). The usual indication for operation, in the large bowel, is stricture formation but occasionally uncontrollable large bowel disease occurs. If the rectum is normal, colectomy and ileo-rectal anastomosis is performed but the results are rather disappointing with about 50 per cent recurrence within five years. If the rectum is involved, panproctocolectomy and ileostomy is the operation of choice. After this operation, only about seven per cent have recurrent disease and all the recurrences are in the terminal ileum.

\section{Perianal disease}

This is fairly common in Crohn's (25 per cent) and particularly when the colon is involved (75 per cent). Although perianal lesions of fissure, fistula and skin tags look awful they give remarkably little trouble. One must be firm and avoid aggressive surgical itreatment. To know when to operate is always a test of judgement, experience and wisdom. Surgery is sometimes indicated, usually simple drainage for abscesses and no more. Stenosis should be treated by very gentle dilatation over a prolonged period of time. Incontinence is difficult to treat and, if severe, a proctectomy is indicated.

\section{The results of surgical treatment for Crohn's disease}

Reviews over a duration of 20 years show that about 85 per cent of survivors are in good general health. The role of surgery must be placed within the natural history of the disease, the eternal balance must be weighed-on the one hand conservative treatment is relatively safe, and on the other hand surgery is associated with an immediate risk to life of about three to five per cent. On one side, long term conservative management does little to alleviate symptoms, on the other side, surgery is notably successful in this respect. Surgical treatment is also divided into Radical and Conservative (Table II). Judgement has to be reached. Are the

Table II

Radical and conservative surgery (After Alexander-Williams ${ }^{10}$ )

\begin{tabular}{|l|c|c|}
\hline & Leeds (radical) & Birmingham (conservative) \\
\hline Number of patients & 295 & 254 \\
\hline Operations & 415 & 677 \\
\hline Mean operations/patient & 1.4 & 2.7 \\
\hline Surgical deaths & 24 & 15 \\
\hline Surgical mortality & 8.1 per cent & 5.9 per cent \\
\hline Operative mortality & 5.8 per cent & 2.2 per cent \\
\hline
\end{tabular}

patient's symptoms sufficiently disabling to justify recommending, and informed acceptance of, an immediate risk to life of about three per cent? 
If I had to summarise what I have said in one sentence let it be that "the surgeon should sign a tripartite non-aggression pact in Crohn's disease. The three parties being the patient, his physician and his surgeon".

\section{Ulcerative colitis}

Effective management of Ulcerative colitis requires close co-operation between physician and surgeon. I believe that the surgeon should see all severe cases early so that he knows about them beforehand, and that all the current acute and selected chronic cases should be presented to a joint committee. In the Royal Army Medical Corps we do this at our combined gastro-enterology conferences held weekly at the Queen Elizabeth Military Hospital and the other Services do likewise.

The majority of patients with Ulcerative colitis have distal disease of mild or moderately severe and for them the need for surgery is uncommon.

\section{Severe acute ulcerative colitis}

\section{Complications of ulcerative colitis}

The more serious complications of fulminating colitis are: a. Perforation. $b$. Acule toxic dilatation of the colon. $c$. Severe bleeding.

Any of these complications is an indication for emergency total colectomy following adequate resuscitation.

Perforation is most common is severe total colon disease usually during the course of the first attack. If the patient is on steroid therapy the diagnosis may be masked and the physical signs vague, even though the patient is acutely ill, but in the majority the patients develop severe pain and generalised abdominal rigidity so that the diagnosis can readily be made.

During an acute attack there may be toxic dilatation of the colon, which is often difficult to distinguish from perforation. The patient collapses, becomes mentally confused and the abdomen rapidly distends. Both these conditions may be diagnosed from plain abdominal radiographs which should be performed in all cases of severe colitis. The combination of dilatation and marked mucosal irregularity suggests that there is partial denudation of mucosa with spread of inflammation into the deep muscle layers. The distention is usually worse in the ascending and transverse colon. The outlook is serious because mortality for this condition is between 10 and 30 per cent but of all colectomies for Ulcerative colitis about 20 per cent have been required for this condition.

Severe haemorrhage is the indication for operation in about 50 per cent of colectomies. In the majority, profuse bleeding settles with conservative treatment but occasionally an emergency total colectomy is necessary.

These three are absolute indications for urgent operation, sometimes without preliminary drug treatment. Usually they are the threat that lurks behind the management of severe colitis requiring potent drug therapy. The better indication for urgent surgery is the failure to respond to such medical treatment, for we are attempting to avoid the more serious complications described, by good judgement in performing early surgery. Useful indications of failure to respond are the development or persistence of high fever or pulse rate, anorexia and malaise, severe 
abdominal tenderness, fall in body weight, haemoglobin and serum albumin. The timing of operation varies according to the clinical condition of the patient, if there is no improvement, a decision is usually reached within a few days after admission to hospital. If there is some improvement, the decision to delay may be delayed for a week or two. The mortality, after operation, in elderly patients is particularly high. Therefore early, rather than late, surgical treatment for those over 60 years of age should be the rule.

Mild distal colitis rarely requires surgical treatment. If it is confined to the reach of the sigmoidoscope there is only a two per cent chance of operation being required within five years, and only a five per cent chance of the disease spreading to the splenic flexture (Ritchie $e t a l^{4}$ ).

\section{Chronic relapsing colitis}

Is the commonest indication for total colectomy. The mortality in this group for elective, well prepared, patients is about two to three per cent and the morbidity is correspondingly lower than for emergency colectomy. It is for this reason that operation should, ideally, be performed as a planned procedure.

Carcinoma of the colon may complicate chronic Ulcerative colitis. The incidence of malignancy increases with the duration of the disease and with the extent of involvement of the colon. At least 30 per cent of all cases lasting twelve years develop carcinoma, commonly multi-focal. The clinical diagnosis may be difficult because the symptoms of carcinoma closely resemble an exacerbation of colitis. Those patients most at risk are those where there was a clinically severe first attack, pan-proctocolitis, chronic continuous symptoms and where the onset of colitis was in childhood or early adult life.

\section{Surgical management}

There are three basic techniques for the treatment of Ulcerative colitis:

a. Pan-proctocolectomy with ileostomy. b. Colectomy and ileo-rectal anastomosis. c. Colectomy, ileostomy and rectosigmoid mucous fistula.

Each procedure of these operations has its own problems technically and its complications. Whenever the colon or rectum is handled or cut we accept the very real risk of wound infection. Whenever an ileostomy is fashioned we accept its attendant problems, similarly with a colon anastomosis. When the rectum is excised, we accept the extra risk of sexual dysfunction due to damage of the autonomic nerves and the problems of persistent sinuses. All these risks and complications of surgery, both in general and specific, have to be weighed against the risks in a particular patient of leaving their colon intact. All physicians will have experienced the feeling that the surgeon is dragging his feet sometimes, but when he has been invited to see the results of complications after operation, he will realise why some wisdom in leaving a patient alone is required. Such wisdom, of when not to operate, is always acquired the hard way-hard on the patient that is -and we must instil this wisdom into both surgeon and physician by utilising other clinicians experience and by studying the written word. This is why close collaboration is so healthy for us and is the main purpose of this symposium today. 
The management may be conveniently divided into the acute attacks with an emergency operation, and the chronic disease with an elective operation.

\section{In acute attack}

a. Transvierse colostomy in fulminating cases where the patient is too ill for colectomy. b. Colectomy, ileostomy and rectosigmoid mucous fistula: (i) With secondary excision of the rectum when the patient's condition permits. (ii) With later conversion to ileo-rectal anastomosis. c. Pan-proctocolectomy with ileostomy.

Sepsis complicates operations performed in the presence of free perforation or massive dilatation of the collon. In the latter, the wall of the bowel resembles the texture of blotting paper and tends to tear without any warning, discharging its highly infected and toxic contents into the peritoneal cavity. This is one of the reasons for the relatively high mortality for such emergency operations. The operation can be very difficult indeed and this is when the experience, judgement, technique and speed of the surgeon are the vital faotors in success.

\section{In chronic disease}

In chronic disease the elective operations are :

a. Pan-proctocolectomy with ileostomy. b. Colectomy with ileo-rectal anastomosis (with or without temporary ileostomy). c. Sub-total colectomy with caecoproctostomy.

I have put pan-proctocolectomy and ileostomy first because it is an effective and satisfactory treatment for Ulcerative colitis when the criteria for operation have been satisfied. It was probably the first operation most of us saw, that actually cured very ill, debilitated and worn out patients. The removial of the colon often results in a dramatic improvement so that the day after operation a patient with all the paraphernalia of surgery on the colon-a painful cut on the belly, another cut on his perineum, drips, tubes, ileostomy and bag-may beam at you and say how well he is feeling. Providing his ileostomy functions well and the perineum heals, the patient, the physician and the surgeon are all happy. It is difficult not to regard this as the basic result and weigh up alternative methods against this standard.

Such an operation is colectomy with ileostomosis and this operation is particularly associated with Stanley Aylett ${ }^{5}$ and the Gordon Hospital. His figures for 369 cases in 1952 to 1968 showed well over 80 per cent were restored to normal health. The operative mortality was five per cent overall including 14 per cent of 55 fulminating and three per cent of 142 chronic cases. He claimed that the inflammatory changes in the rectum resolved in most cases. Most other surgeons have not been able to achieve such good results and the St Mark's series had a 50 per cent recurrence rate associated with failure of the inflammatory changes in the rectum to resolve. This experience is common.

The risk of developing carcinoma in the rectum is ever present and is related to the control of the disease. Careful follow-up and regular biopsies are mandatory. I believe the operation should be reserved for patients with favourable signs. That is, those with a clear rectum or with minimal disease, who can attend for regular follow-up and who are prepared to have a second major operation if necessary. 
Other more recent developments in surgical techniques include the following: Sub-total colectomy with caecoproctostomy (Webster and Howard ${ }^{6}$ ).

\section{Reservoir or continent ileostomy}

The development of a reservoir ileostomy which can be emptied periodically by catheter has been an important contribution by Koch. Problems with the construction of a valve have still not been completely solved, other techniques using a magnetic closing device have also been developed.

\section{Colectomy and ileo-anal pull-through}

An anastomosis after coning out the mucosa of the ano-rectal remnant is another technique which, although tedious, has had promising results.

Colectomy, ileal reservoir and ileo-anal pull through anatomosis has recently been successful in the hands of Sir Alan Parkes ${ }^{7}$ in some nine cases.

Two major points of technique are worth attention, they are the formation of the ileostomy and the excision of the rectum. The site for ileostomy should be marked before operation so that the appliance fits correctly and can be seen and managed by the patient. The ileum must be brought out through a rectus split incision, which takes two finger comfortably, the bowel must not be twisted, the mesentery should lie facing the head and should be carefully sutured to the peritoneum to avoid potential internal hernia. About $6 \mathrm{~cm}$ of ileum should be drawn through onto the surface of the abdomen and it must be sutured in such a way that the outer $3 \mathrm{~cm}$ is everted over the inner layer to form a spout, with mucosa to skin apposition. One hopes to avoid ileostomy stenosis, regression or prolapse, skin excoriation internal and para-ileostomy hernia.

Excision of the rectum should be done by a perimuscular approach (Lee and Dowling ${ }^{8}$ ) because this is not a cancer openation and tissues outside the rectum need not be taken. The levators should be cut away from the rectum and the plane desired is just outside the bowel wall. This technique avoids damage to the nervi erigentes and consequent sexual problems such as sterility or impotence.

Local complications of ulcerative colitis

Fissures, oedematous skin tags and fistula in ano all occur in Ulcerative colitis but much less than in Crohn's disease.

\section{Summary of surgical treatment}

Pan-proctocolectomy is an effective operation that cures Ulcerative colitis at the price of a permanent ileostomy. The operation has a mortality that is low (iwo to five per cent) in elective but higher (10 to 30 per cent) in emergency operations $\left(\mathrm{Ritchi}^{\mathrm{9}}\right.$ ). It also has a considerable postoperative morbidity with complications such as intestinal obstruction and there is a small but definite risk of sexual dysfunction in males. The majority of patients with an ileostomy adapt well and live normal, healthy, active lives.

There are a number of patients who find an ileostomy a great disability either because of their fastidious personality or because the stoma is technically unsatisfactory. Colectomy with ileo-rectal anastomosis is an attractive alternative to proctocolectomy and ileostomy but this operation also has problems. Ulcerative colitis almost always involves the rectum and this operation removes the involved 
colon but commonly leaves an inflamed rectum. Persisting or recurrent proctitis is common and further medical treatment is of benefit and may well keep it under control. The patient's general health might well be restored by removal of the colon but symptoms of diarrhoea and urgency may persist, due to liquid or semiformed ileal effluent entering an inflamed, sometimes narrowed, rectum. There is pensistence of the increased risk of carinoma in the diseased rectum and careful follow-up with regular mucosal biopsy is necessary. The avoidance of a stoma and the preservation of sexual function in males outweigh the disadvantages of the procedure and certainly the normal four or five loose bowel actions a day and regular sigmoidoscopy is considered by most patients to be a small price to pay. We might, therefore, regard colectomy and ileo-rectal anastomosis as an operation to be done in highly selected cases and is particularly suitable for younger patients provided there is no severe rectal or perianal disease. It has also a lot to recommend it for patients of all ages when the rectum is normal or only slightly diseased. It is essential that before recommending a patient to accept ileo-rectal anastomosis he should have explained to him and should understand the arguments for and against the procedure. He should be warned that a perfect functional result is not to be expected and that some bowel frequency will persist, some medical treatment for diarrhoea or rectal inflammation may be needed and that indefinite supervision as an outpatient is necessary. A patient who has the pros and cons explained to him and who accepts operation after making an informed judgement will be delighted with an excellent result, accept the average and will be more philosophical about a poor result and the prospect of a further operation.

If a patient demands a certain cure of colitis in one operation or if the patient is in the older age group so that a cure by one operation is indicated clinically, or if the rectum or anus is severely diseased, or if there appears to be a major carcinoma risk then pan-proctocolectomy as a primary procedure is to be preferred. It is arguable as to whether or not all patients with chronic Uicerative colitis should be advised to undergo surgical treatment. When meticulous medical supervision is possible, particularly at a centre that specialises in the treatment of this disease, then the results suggest that it is safe to allow a proportion of such patients to continue without operation. Where regular follow-up is suspect, where there is chronic disability or where there is evidence of dysplasia in the mucosal biopsy, operation should always be advised.

\section{REFERENCES}

1. Steinberg, D M et al (1972). Gut 14, 865

2. KYLE, J (1971). British Journal of Surgery 58, 735

3. 'Lockhart-Mummery, H E (1972). British Journal of Surgery 59, 823

4. Ritchie, J K, Powell-Tuck, J and LenNard-Jones, J E (1978). Lancet i, 1140

5. Aylett, S (1971). Proceedings of the Royal Society of Medicine 64, 967

6. WeBSTER, C V and Howard, R, R S (1973). British Journal of Surgery 60, 42

7. PARKes, A (1978). British Medical Journal iii, 85-88

8. LeE, E C G and Dowling, B L (1972). British Journal of Surgery 59, 29

9. Ritchie, J K (1971). Gut 12, 528-536

10. Alexander-Williams, J (1972). Clinics in Gastroenterology 1, 469 